05,10

\title{
Электроакустическое возбуждение спиновых волн и их детектирование за счет обратного спинового эффекта Холла
}

\author{
() Н.И. Ползикова, С.Г. Алексеев, В.А. Лузанов, А.О. Раевский \\ Институт радиотехники и электроники им. В.А. Котельникова РАН, \\ Москва, Россия \\ E-mail: polz@cplire.ru
}

\begin{abstract}
Представлены теоретические и экспериментальные результаты по возбуждению и детектированию спиновых волн и спиновых токов в многочастотных резонаторах объемных акустических волн, содержащих пленки железоиттриевого граната (YIG) в контакте с пленкой Pt. Установлено, что акустически возбужденная магнитная прецессия в условиях резонатора создает спиновою накачку, которая также носит резонансный характер. Выявлена существенная асимметрия частотной зависимости сигнала напряжения, детектируемого на пленке $\mathrm{Pt}$, относительно частоты магнтоупругого резонанса, которая подтверждается теоретическими расчетами. Показано, что комбинация метода акустической резонаторной спектроскопии в сочетании с электрическим детектированием магнитной динамики с помощью обратного спинового эффекта Холла представляет интерес для изучения дисперсии спиновых волн, а также магнитных и магнитоупругих параметров ферромагнитных пленок.
\end{abstract}

Работа выполнена при частичной поддержке грантов РФФИ № 16-07-01210, № 17-07-01498.

DOI: 10.21883/FTT.2018.11.46659.17NN

\section{1. Введение}

Как было показано еще в конце 1950-х годов, при фазовом синхронизме акустической (AW) и спиновой волны (SW), то есть в условиях магнитоупругого резонанса (MER), происходит образование связанных магнитоупругих (ME) волн [1-3], которые в настоящее время все чаще называют магнон-поляронами [4]. МЕ-волны образуются, как при условиях возбуждения магнитной прецессии, так и при возбуждении AW различными пьезоэлектрическими преобразователями. В последнем случае происходит электроакустическое (без применения высокочастотных магнитных полей) возбуждение и детектирования спиновой динамики, часто называемой акустически возбужденным ферромагнитным резонансом (ADFMR - acoustically driven FMR). При этом под FMR подразумевается скорее неоднородный FMR, т.е., либо спинволновой резонанс (SWR), либо просто бегущие SW. Поэтому мы будем называть акустически возбужденные магнитные колебания ADSW (acoustically driven SWs).

Возобновление интереса к ADSW произошло относительно недавно в связи актуальными задачами магноники, стрэйнтроники и микроволновой спинтроники. Поскольку пьезоэлектрическое возбуждение ADSW не требует создания высокочастотных магнитных полей, предполагается, что устройства на основе ADSW могут работать с низким энергетическим потреблением. Так, в области микроволновой спинтроники ADSW представляют интерес для акустической спиновой накачки (ASP) [5-7] — преобразования спинового углового момента ADSW в ферромагнетике (FM) (например, железо-иттриевом гранате (YIG)) в постоянный спино- вый ток на границе с парамагнитным металлом (NM) (например, с Pt).

Акустическая спиновая накачка была предложена и продемонстрирована в ряде работ, которые делятся на две группы. Так в работах [7-9] наблюдалась нерезонансная ASP в структуре с YIG/Pt. B [5] было экспериментально продемонстрирована генерация спиновых токов в результате резонансного взаимодействия магнонов и когерентных фононов поверхностной акустической волны (SAW) в двуслойной тонкопленочной структуре $\mathrm{Co} / \mathrm{Pt}$. Нерезонансная накачка в работах [7-9] осуществлялась на частотах в несколько мегагерц, что существенно меньше частот FMR, в то время как резонансная накачка в [5] осуществлялась на частотах SAW в гигагерцовом диапазоне, совпадающих с частотами ADSW.

Для генерации ADSW чаще всего используются SAW, возбуждаемые встречно-штыревыми преобразователями [5,8,10-14]. Технология SAW хорошо разработана, но имеет существенные ограничения на частотах превышающих 2.5-3 GHz в связи с требованиями к фотолитографии. Для создания эффективной ASP недавно нами был предложен композитный магнитоэлектрический спинтронный резонатор объемных акустических волн (BAW), работающий на высоких гармониках - (HBAR - High overtone Bulk Acoustic wave Resonator) $[15,16]$.

Преимущество использования HBAR со структурой YIG/Pt обусловлено тем, что в гигагерцовом диапазоне он сравнительно легко возбуждается на низкой гармонике пьезопреобразователя $(m=1,3)$. При этом высокие гармоники всей структуры $f_{n}(n \sim 500)$, разделенные по частоте всего на несколько мегагерц, имеют высокую добротность $Q \sim 10^{4}$, что позволяет эффективно накапливать упругую энергию для передачи ее в магнитную подсистему при магнитном поле, соответствующем 
MER. K тому же, как было показано нами ранее [17-19], частоты самого резонатора $f_{n}$, очень чувствительны относительно изменения поля вблизи MER, что позволяет однозначно определить связь между сигналами напряжения на платине, создаваемые комбинацией ASP и обратного спинового эффекта Холла (ISHE) [20], и возбуждением SW в YIG. B нашей недавней работе [21] мы получили величину напряжения $U_{\text {ISHE }}$ в микровольтовом диапазоне при подаваемой на электроды пьезопреобразователя мощности $\sim 2 \mathrm{~mW}$ и активной области возбуждения $\sim 170 \mu \mathrm{m}$. В то же время была обнаружена значительная асимметрия интенсивности $U_{\text {ISHE }}$ относительно частоты $f_{\text {MER }}(H)$. Объяснение этой асимметрии было дано на основе качественного рассмотрения дисперсионных характеристик, связанных МЕ-волн. Отсутствие теории, описывающей возбуждение ASP в BAW резонаторе явилось мотивацией настоящей работы. Наиболее близкой к нашей задаче представляется работа [22], однако в ней рассмотрен только ферромагнитный слой, граничащий с бесконечной упругой средой.

В настоящей работе строится теоретическая модель, описывающая генерацию ADSW в пленке YIG с помощью пьезоэлектрического преобразователя в условиях двойного резонанса: магнитоупругого резонанса в YIG и чисто упругого во всей многослойной структуре BAW резонатора. В результате последовательного вычислении акустических импедансов всех слов резонаторной структуры, выводится аналитическое выражение для электрического импеданса преобразователя. Эта характеристика напрямую связана с комплексным коэффициентом отражения, что позволяют проводить сравнение с экспериментальными данными по наблюдению особенностей возбуждения и детектирования ADSW. На основе решения уравнений движения во всех слоях структуры теоретически рассчитывается эффективность генерации SC на границе $\mathrm{YIG} / \mathrm{Pt}$ методом резонансной ASP. Полученные частотные зависимости напряжения ISHE сравниваются с экспериментальными. Дается обоснование выявленной в работе [21] существенной асимметрии частотной зависимости сигнала напряжения относительно частоты MER.

\section{2. Постановка задачи и основные уравнения}

На рис. 1 показана структура магнитоэлектрического резонатора высоких гармоник объемных акустических волн (HBAR) для электроакустического возбуждения и детектирования спиновых волн в FM-слоях и создания спиновой накачки: генерации SC на границе FM с парамагнитным металлом. Элементы резонатора показаны на рис. 1 цифрами $(1-5)$.

Резонатор содержит FM-пленки YIG (3 и 4) на подложке (5) из GGG. Пленки YIG намагничены до насыщения $M_{0}$ во внешнем магнитном поле $H_{0}$, приложенном в плоскости структуры. На одной из плоскостей

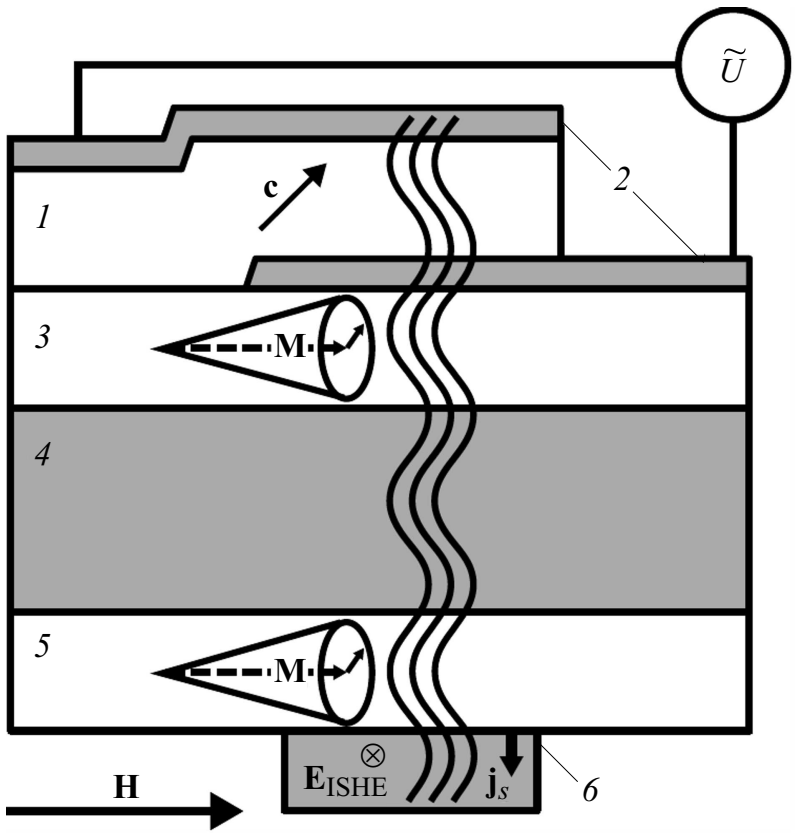

Рис. 1. Схема резонатора: 1 - пленка $\mathrm{ZnO}$ толщиной $l_{1}=3 \mu \mathrm{m}, 2$ - электроды из $\mathrm{Al}$ толщиной $l_{2}=150-200 \mathrm{~nm} \mathrm{c}$ диаметрами в области перекрытия $a=170 \mu \mathrm{m}, 3,5$ - эпитаксиальные пленки YIG с толщинами $l_{3,5}=s=30 \mu \mathrm{m}, 4-$ монокристаллическая подложка из GGG толщиной $l_{4}=500 \mu \mathrm{m}$, 6 - пленка Pt толщиной $l_{6}=12 \mathrm{~nm}$, сформированная в виде полосы с направлением а перпендикулярным плоскости структуры. Пьезоэлектрическая ось с составляет угол $30^{\circ}-40^{\circ}$ относительно нормали к плоскостям структуры.

структуры находится тонкопленочный пьезоэлектрический преобразователь из пьезоэлектрика $\mathrm{ZnO}$ (1) с двумя пленочными электродами из Al (2), который служит для возбуждения и приема поперечных акустических волн в гигагерцовом диапазоне частот, распространяющихся перпендикулярно слоям. Возбуждение именно поперечных волн принципиально, поскольку в данной геометрии именно поперечные волны с вектором поляризации коллинеарным полю взаимодействуют с магнитной подсистемой при любой взаимной ориентации поля и кристаллографических осей YIG. Структура может содержать как один слой магнетика, например, эпитаксиальную пленку YIG на подложке из галлий-гадолиниевого граната (GGG), как показано на рис. 1, так и несколько слоев. Поскольку экспериментальные структуры содержали эпитаксиальные пленки YIG, выращенные с двух сторон подложки, то мы также будем рассматривать такие двусторонние структуры.

Вследствие ME-взаимодействия в слоях YIG возбуждаются магнитные колебания - ADSW. Из-за обратного влияния ADSW на акустическую подсистему YIG возможно их опосредованное детектирование с помощью электроакустического преобразователя по изменению частотной зависимости входного электрического импеданса $Z_{e}$ под действием магнитного поля $[18,19]$. 
Наиболее сильно эффект взаимодействия должен проявляться в условиях MER, частоты которого определяются величиной внешнего магнитного поля, магнитными и упругими параметрами YIG.

На нижней пленке YIG (5) находится пленка немагнитного металла (6), в которую инжектируется спиновый ток. Таким образом, измеряя сигнал постоянного напряжения, обусловленный конвертацией спинового тока в ток проводимости за счет ISHE [20], можно непосредственно детектировать ADSWs и создаваемые ими спиновые токи.

\section{1. Уравнения для магнитоупругой среды}

Для получения связанных линейных динамических уравнений движения для упругого смещения и и уравнения прецессии намагниченности $\mathbf{m}$ МЕ-энергия должна быть записана с точностью до квадратичных членов по малым переменным. Будем считать, что МЕ-связь обусловлена линейной магнитострикцией, возникающей вследствие зависимости энергии магнитной кристаллической анизотропии от тензора деформации $u_{i j}$ [2]. Тогда в главных осях кубического ферромагнетика плотность ME-энергии имеет вид

$$
W_{\mathrm{ME}}=B_{1} u_{i i} v_{i}^{2}+B_{2} u_{i k} v_{i} v_{k}\left(1-\delta_{i k}\right),
$$

где $B_{1,2}-$ магнитоупругие константы, $v_{i}-$ направляющие косинусы вектора намагниченности, $\delta_{i k}-$ символ Кронеккера. Зависимость всех переменных величин от координат и времени выбираем в виде $\exp i(k x-\omega t)$, где $k$ и $\omega-$ соответственно волновое число и частота волны, $x$-координата в направлении распространения волн. Внешнее магнитное поле $\mathbf{H}_{0}$, параллельное оси $z$, создает в ферритовом слое намагниченность насыщения $\mathbf{M}_{0} \| \mathbf{H}_{0}$. Связанная система уравнений для поперечной компоненты упругого смещения $u_{z}=U$ и переменной намагниченности $\mathbf{m}=\left(m_{x}, m_{y}\right)$ имеет вид

$$
\begin{gathered}
\left(\omega^{2}-V^{2} k^{2}\right) U=i\left(b / \rho M_{0}\right) k m_{x}, \\
i \omega m_{x}=\omega_{H} m_{y}, \quad i \omega m_{y}=\left(\omega_{H}+\omega_{m}\right) m_{x}+i \gamma k b U .
\end{gathered}
$$

Здесь $V=\sqrt{C / \rho}-$ фазовая скорость поперечной BAW в среде с плотностью $\rho$ и упругим модулем $C=C_{44}, b=B_{2}, \gamma-$ гиромагнитное отношение, $\omega_{H}=\omega_{H}\left(k^{2}\right)=\gamma\left(H+D k^{2}\right), \omega_{m}=\gamma 4 \pi M_{0}, D-$ обменная константа, $H=H_{0}+H_{d}+H_{a}$ - постоянная составляющая эффективного магнитного поля, $H_{d}, H_{a}-$ поля размагничивания и анизотропии. Как следует из (1), в данной геометрии другие компоненты смещения (продольная и поперечная) не испытывают влияния намагниченности и наоборот. Уравнения (2) получены в магнитостатическом приближении для уравнений Максвелла, из которых находится поле размагничивания $\mathbf{h}^{m}=\left(-4 \pi m_{x}, 0,0\right)$. Отличная от нуля компонента тензора напряжений имеет вид

$$
T_{z x}=C \frac{d U}{d x}+\frac{b}{M_{0}} m_{x} .
$$

Из (2) можно получить дисперсионное уравнение для связанных волн

$$
\left(\omega^{2}-k^{2} V^{2}\right)\left(\omega^{2}-\omega_{0}^{2}\right)-\xi \omega_{H} \omega_{M} k^{2} V^{2}=0,
$$

где $\xi=b^{2} /\left(4 \pi C M_{0}^{2}\right)$ - безразмерная эффективная константа МЕ-связи. Уравнение (4) является биквадратным относительно частоты и легко может быть решено. Фононная ветка имеет вид $\omega=\omega_{\mathrm{AW}} \approx V \operatorname{Re} k$. Магнонное решение $\omega=\omega_{\mathrm{SW}} \approx \omega_{0}\left(k^{2}\right)$ состоит из двух ветвей. Одна из них всегда проходит в области мнимых волновых чисел и соответствует эванесцентному решению для прецессии намагниченности с левой поляризацией вращения вектора намагниченности $[23,24]$. Вторая ветвь соответствует правой поляризации и является распространяющимся решением при $\omega>\omega_{0}(0)$ и эванесцентным при $\omega<\omega_{0}(0)$.

Для нахождения решения граничной задачи уравнение (4) нужно разрешить относительно волнового числа $k$. Поскольку это уравнение является бикубическим, оно имеет шесть корней $k=k_{n}$, причем $k_{2 i}=-k_{2 i-1}$, где $i=1,2,3$. При заданной частоте три парциальных корня соответствуют вышеописанным распространяющимся и эванесцентным решениям с правым и левым направлением вращения намагниченности. Корни $k_{n}$ можно найти в явном виде, например, по формулам Кардано, после чего можно записать общее решение (3) и (4) в форме

$$
\left(\begin{array}{c}
U \\
m_{x} \\
m_{y} \\
T_{z x}
\end{array}\right)=\sum_{n=1}^{6}\left(\begin{array}{c}
1 \\
\beta_{n} \\
\delta_{n} \\
\alpha_{n}
\end{array}\right) A_{n} \exp \left(i k_{n} x\right)
$$

где амплитудные коэффициенты имеют вид

$$
\begin{gathered}
\beta_{n}=i \gamma b \beta_{n}^{0}, \quad \delta_{n}=\frac{i \omega}{\omega_{H}\left(k_{n}\right)} \beta_{n}, \\
\alpha_{n}=-i C k_{n}+i \gamma \frac{b^{2}}{M_{0}} \beta_{n}^{0}, \quad \beta_{n}^{0}=\frac{k_{n} \omega_{H}\left(k_{n}\right)}{\omega_{0}^{2}\left(k_{n}\right)-\omega^{2}} .
\end{gathered}
$$

На границах магнитоупорядоченных слоев решение (5) должно удовлетворять граничным условиям: электродинамическим, упругим, а также условиям на намагниченность. Далее координаты нижних поверхностей слоев с индексом $i$ будем обозначать как $x_{i}$, толщины слоев - как $l_{i}$.

В принятых приближениях электродинамические граничные условия удовлетворяются автоматически ввиду отсутствия переменных тангенциальных полей и нормальных индукций. Упругие условия сводятся к непрерывности упругих смещений и нормальных напряжений. Для намагниченности будем рассматривать случай свободных спинов, полагая равенство нулю производных от переменной намагниченности на границах FM-пленок. 


\section{2. Уравнения для немагнитных сред}

В слоях немагнитных диэлектриков с индексом $i$ решения для упругих смещений и напряжений представим в виде

$$
\begin{aligned}
U^{(i)}= & U^{+(i)} \exp \left(i k^{(i)} x\right)+U^{-(i)} \exp \left(-i k^{(i)} x\right), \\
T_{z x}^{(i)}= & i k^{(i)} C^{(i)}\left[U^{+(i)} \exp \left(i k^{(i)} x\right)\right. \\
& \left.-U^{-(i)} \exp \left(-i k^{(i)} x\right)\right]+\frac{4 \pi e}{\varepsilon} D_{0} \delta_{1 i} .
\end{aligned}
$$

Здесь $k^{(i)}=\omega / V^{(i)}, V^{(i)}=\sqrt{C^{(i)} / \rho^{(i)}}, \rho^{(i)}, C^{(i)}$ - волновые числа, скорости, плотности и эффективные упругие модули (с учетом пьезоэффекта в слое 1) в каждой из сред, $e-$ пьезоэлектрическая константа, $D_{0}-$ однородная электрическая индукция в пьезоэлектрике. Последняя связана с протекающим переменным током: $I=S \frac{\partial D_{0}}{\partial t}=-i \omega S D_{0}$, где $S-$ площадь электродов преобразователя. Электрическое напряжение $\tilde{U}$, возникающее между плоскостями пьезоэлектрика $x=x_{1}$ и $x=x_{1}+l_{1}$, можно представить как

$$
\tilde{U}=\frac{e}{\varepsilon}\left[U^{(1)}\left(x_{1}\right)-U^{(1)}\left(x_{1}+l_{1}\right)\right]+\frac{I}{i \omega C_{0}},
$$

где $C_{0}$ - емкость пьезоэлектрического слоя.

\section{3. Вычисление входного электрического импеданса преобразователя}

Далее мы применим методику, основанную на последовательном вычислении входных акустических импедансов всех слов резонаторной структуры. Определим входной акустический импеданс слоя с индексом $i$ как

$$
z_{\text {in }}^{(i)}=T_{z x}\left(x_{i}+l_{i}\right) /\left(d U\left(x_{i}+l_{i}\right) / d t\right) .
$$

Считаем, что механические нагрузки на пленку пьезоэлектрика, создаваемые металлическими электродами $\left(l_{2} \sim 150-200 \mathrm{~nm}\right)$ и воздухом, а также пленкой $\mathrm{Pt}$ $\left(l_{6} \sim 12 \mathrm{~nm}\right)$ на нижней поверхности структуры, пренебрежимо малы.

В случае немагнитных диэлектрических слоев импедансы (9) вычисляется с помощью последовательного применения формулы трансформации импеданса для каждого слоя

$$
z_{\text {in }}^{(i)}=z^{(i)} \frac{Z_{L}^{(i)} \cos \theta_{i}+i z^{(i)} \sin \theta_{i}}{z^{(i)} \cos \theta_{i}+i Z_{L}^{(i)} \sin \theta_{i}}, \quad Z_{L}^{(i)}=z_{i n}^{i+1} .
$$

Здесь и далее $z^{(i)}=\rho^{(i)} V^{(i)}$ и $\theta_{i}=k^{(i)} l_{i}$ - материальные акустические импедансы и набеги фазы для соответствующих слоев, $Z_{L}^{(i)}$ - импеданс нагрузки.

Для слоев магнетика выражение (10) неприменимо, поскольку среда не может быть описана единственным волновым числом и, соответственно, набегом фазы. После подстановки решения (5) в соответствующие граничные условия выразим все амплитуды $A_{n}$ через одну, например, через $B_{1}=\left(A_{1}+A_{2}\right) / 2$. Далее, применив соотношение (9), найдем искомый импеданс для FM-слоя с учетом ME-взаимодействия и пространственной дисперсии SW. Для FM-слоя с индексом $i$, толщиной $l_{i}=s$ и нагруженного на импеданс $Z_{L}^{(i)}$ получаем

$$
\begin{gathered}
z_{\text {in }}^{(i)}=\frac{1}{\tilde{\omega}} \frac{2 z_{1} z_{2}+z_{\text {in }}^{(i+1)} \tilde{\omega}\left(z_{1}-z_{2}\right)}{\left(z_{1}-z_{2}\right)-2 z_{\text {in }}^{(i+1)} \tilde{\omega}} \\
z_{1}=\sum_{1}^{3} \alpha_{i} \gamma_{i} \operatorname{tg}\left(k_{i} s / 2\right), \quad z_{2}=\sum_{1}^{3} \alpha_{i} \gamma_{i} \operatorname{ctg}\left(k_{i} s / 2\right), \\
\tilde{\omega}=\omega \sum_{1}^{3} \gamma_{i}, \quad \gamma_{2,3}=\frac{k_{1} \delta_{1}}{k_{2,3} \delta_{2,3}} \frac{\left[k_{1}^{2}-k_{3,2}^{2}\right]}{\left[k_{2,3}^{2}-k_{3,2}^{2}\right]}, \quad \gamma_{1}=1
\end{gathered}
$$

На основе соотношений (9)-(12) вычисляется входной акустический импеданс нагрузки преобразователя

$$
z=T_{z x}\left(x_{2}\right) /\left(d U\left(x_{2}\right) / d t\right)=z_{\text {in }}^{(3)} .
$$

Теперь можно использовать хорошо известное выражение для входного электрического импеданса пьезоэлектрического преобразователя $Z_{e}[25]$ или вывести его, учитывая механические граничные условия и соотношения (7) и (8), в виде

$$
\begin{aligned}
Z_{e} & =\tilde{U} / I=\frac{1}{i \omega C_{0}}\left(1+\frac{K_{t}^{2}}{\theta_{1}} \frac{i z \sin \theta_{1}-2 z^{(1)}\left(1-\cos \theta_{1}\right)}{z^{(1)} \sin \theta_{1}-i z \cos \theta_{1}}\right) \\
& =\frac{1}{i \omega C_{0}}\left(1+Z_{\mathrm{AW}}\right) .
\end{aligned}
$$

Здесь $K_{t}$ - эффективная пьезоэлектрическая постоянная для поперечной BAW в материале пьезопреобразователя. Подстановка формул (9)-(13) в (14) позволяет получить аналитическое выражение для электрического импеданса, которое в дальнейшем анализируется численно.

\section{4. Связь намагниченности с электрическими параметрами преобразователя}

Приведем выражения для компонент переменной намагниченности в FM-слое с $i=5$, граничащим с пленкой $\mathrm{Pt}$ :

$$
\begin{aligned}
\left.\left(\begin{array}{l}
m_{x} \\
m_{y}
\end{array}\right)\right|_{x=x_{5}}= & -i \omega \exp (-i \omega t) \frac{U\left(x_{4}\right)}{\tilde{\omega}\left(z_{1}-z_{2}\right)} \\
& \times \sum_{k, j=1}^{3} M_{k, j}\left(\begin{array}{c}
\alpha_{k} \beta_{j}-\beta_{k} \alpha_{j} \\
\alpha_{k} \delta_{j}-\delta_{k} \alpha_{j}
\end{array}\right)+c . c .
\end{aligned}
$$

Здесь учтены временны́е множители, $M_{k, j}=\gamma_{k} \gamma_{j} \times$ $\times\left[\operatorname{tg}\left(k_{k} s / 2\right)^{2}-\operatorname{tg}\left(k_{j} s / 2\right)^{2}\right] /\left[\operatorname{tg}\left(k_{k} s / 2\right) \operatorname{tg}\left(k_{j} s / 2\right)\right]$, остальные параметры определяются соотношениями (6) и (12). 
Для нахождения угла прецессии намагниченности следует связать упругое смещение на верхней границе пленки $U\left(x_{4}\right)=B_{1} \sum_{1}^{3} \gamma_{i}$ с энергетическим параметром преобразователя, например, напряжением $\tilde{U}(8)$, возникающим между электродами преобразователя.

Далее для упрощения будем считать, структура содержит только одну FM-пленку, т. е., что $U\left(x_{3}\right)=U\left(x_{2}\right)$. Используя соотношения (7), (9), (10) найдем связь между упругими смещениями на верхней и нижней стороне подложки

$$
U\left(x_{4}\right)=U\left(x_{3}\right) z^{(4)} /\left[z^{(4)} \cos \theta_{4}+i Z_{L}^{(4)} \sin \theta_{4}\right] .
$$

Из уравнений для пьезоэлектрического слоя выразим амплитуду смещения на границе с подложкой в виде

$$
\begin{gathered}
U\left(x_{3}\right)=U\left(x_{2}\right)=2 i e \tilde{U} /\left(\omega l_{1} z \tilde{m}\right), \\
\tilde{m}=\left(i z^{(1)} \sin \theta_{1}+z \cos \theta_{1}\right)\left(1+Z_{\mathrm{AW}}\right) /\left[z \sin \left(\theta_{1} / 2\right)^{2}\right],
\end{gathered}
$$

где квадрат модуля $\tilde{m}$ определяет так называемый формфактор [26]. Формулы (16)-(18) определяют связь между упругим смещением на границе FM с подложкой и напряжением на электродах преобразователя.

\section{5. Детектирование ADSW в пленке платины}

Плотность SC, инжектируемого в тонкую пленку $\mathrm{Pt}$, контактирующую с YIG, запишем как $\left.\mathbf{j}_{s} \propto(\mathbf{m} \times \partial \mathbf{m} / \partial t)\right|_{x=x_{5}}[27]$. Усредненный по времени $\mathrm{SC}$, поляризованный по $\mathbf{z}$, и текущий от поверхности YIG в Pt в направлении $\mathbf{n}$, находится как $\mathbf{j}_{s, \mathbf{z}}=\left\langle\mathbf{j}_{s}\right\rangle \propto \theta^{2} \mathbf{n}$, где $\theta-$ угол конуса прецессии намагниченности (см. рис. 1) у границы с Pt:

$$
\theta=\sqrt{\operatorname{Im}\left[m_{x}^{*}\left(x_{5}\right) m_{y}\left(x_{5}\right) / M_{0}^{2}\right]}
$$

Обратный спиновый эффект Холла приводит к разделению зарядов, в результате чего возникает электростатическое поле $\mathbf{E}_{\mathrm{ISHE}} \propto-\theta_{\mathrm{SH}}\left(\mathbf{j}_{s, \mathbf{z}} \times \mathbf{z}\right)=$ $=-\theta_{\mathrm{SH}} \theta^{2}(\mathbf{n} \times \mathbf{z})$. При положительном спиновом угле Холла $\theta_{\mathrm{SH}}$, что характерно для $\mathrm{Pt}$, направление поля должно быть таким, как показано на рис. 1. Сигнал постоянного напряжения зависит от направления оси полосы платины и находится как

$$
U_{\mathrm{ISHE}}=-\left(\mathbf{E}_{\mathrm{ISHE}} \times \mathbf{a}\right) \propto \theta_{\mathrm{SH}} \theta^{2}((\mathbf{n} \times \mathbf{z}) \cdot \mathbf{a}) .
$$

Здесь считается, что вектор а направлен вдоль полосы платины в направлении от Lo контакта к $H i$ контакту вольтметра [28] и имеет длину, равную активной области возбуждения ADSW, т.е. величину порядка диаметра апертуры преобразователя. Таким образом, измеряя сигнал постоянного напряжения можно детектировать ADSWs и создаваемые ими SCs.

\section{6. Экспериментальная структура и сравнение с теорией}

Экспериментальная структура HBAR (см. рис. 1) была изготовлена на основе коммерческой структуры, состоящей из монокристаллической подложки из GGG c ориентацией (111), на которой с двух сторон методом жидкофазной эпитаксии были выращены пленки YIG, легированные $\mathrm{La}$ и $\mathrm{Ga}$, характеризующиеся пониженной намагниченностью $4 \pi M_{0} \sim 900 \mathrm{G}$. Интерес к легированным пленкам YIG с пониженной намагниченностью обусловлен стремлением избежать нелинейных процессов, влияющих на магнитоупругое возбуждение, которые весьма затруднили бы интерпретацию эксперимента. Поскольку верхняя граница трехмагнонных процессов по частоте определяется соотношением $f=(2 / 3) \gamma 4 \pi M_{0}$, то для чистого YIG с $4 \pi M_{0}=1750 \mathrm{G}$ она составляет $3.25 \mathrm{GHz}$, для образца с $4 \pi M_{0}=350 \mathrm{G}-0.7 \mathrm{GHz}$, а для данного образца $-1.7 \mathrm{GHz}$. На одну из поверхностей структуры наносился тонкопленочный преобразователь. Толщины слоев приведены в подписи к рис. 1. Для возбуждения поперечных $\mathrm{BAW}$ пленки $\mathrm{ZnO}$ с осью текстуры с, составляющей угол $30^{\circ}-40^{\circ}$ с нормалью к плоскости пленки, наносились методом магнетронного распыления. На нижнюю поверхность пленки YIG методом магнетронного напыления наносилась пленка $\mathrm{Pt}$ толщиной $12 \mathrm{~nm}$, которая затем формировалась в виде полосы вдоль вектора a, проходящей непосредственно под пьезопреобразователем. На электроды преобразователя подавалась мощность $2 \mathrm{~mW}$, активная область возбуждения HBAR (область перекрытия электродов) составляла $170 \mu \mathrm{m}$. В диапазоне $3 \mathrm{GHz}$ резонатор возбуждается на третьей гармонике пьезопреобразователя, при этом высокие гармоники $(n \sim 500)$ всей структуры, разделенные по частоте всего на $3 \mathrm{MHz}$, имеют высокую добротность, что позволяет эффективно накапливать упругую энергию для передачи ее в магнитную подсистему. Подробнее технология изготовления структуры спинтронного HBAR, а также экспериментальные методы исследования частотного спектра HBAR и напряжения ISHE в Pt описаны в [15].

Частотные зависимости комплексного коэффициента отражения резонатора $S_{11}$ измерялись в широком диапазоне частот от 0.5 до $4 \mathrm{GHz}$ в касательных к пленке внешних магнитных полях от 0 до $1 \mathrm{kOe}$. Измерения проводились при наиболее выгодной ориентации в плоскости пленки для наблюдения ISHE (20): строго перпендикулярно полосе Pt. В полуавтоматическом режиме были найдены частоты каждого отдельного резонанса $f_{n}$ и их изменение по отношению к частоте резонанса в нулевом магнитном поле. В результате были получены квазинепрерывные зависимости резонансных частот от магнитного поля, приведенные на рис. 2 (точки). Отчетливо видна область MER, локализованная вдоль штрихпунктирной линии, $f_{\text {MER }}(H)$. Положение линии соответствует условиям пересечения невзаимодействующих AW и SW дисперсионных ветвей (5) при 


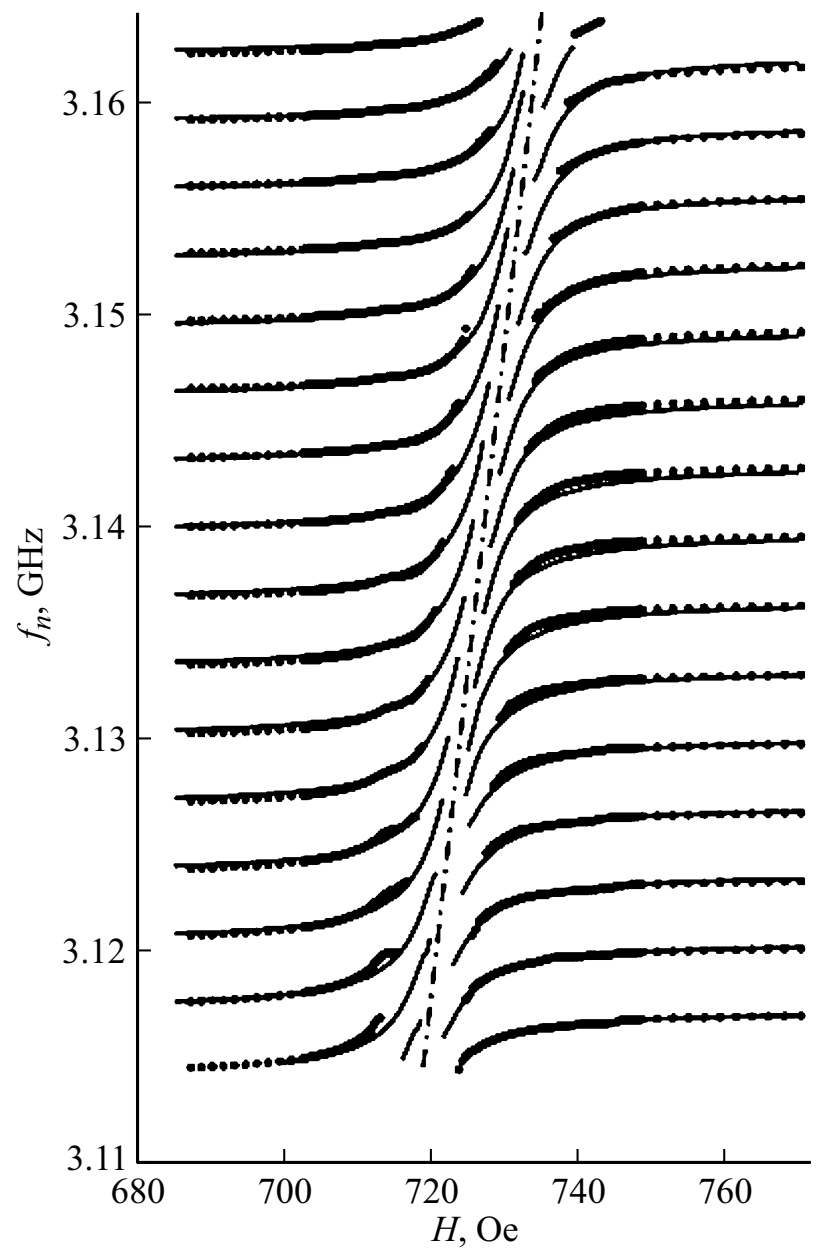

Рис. 2. Зависимость резонансных частот $f_{n}$, от магнитного поля $H$. Сплошные линии - результаты расчета с эффективной магнитоупругой константой $b=4.2 \cdot 10^{6} \mathrm{erg} / \mathrm{cm}^{3}$, кружки - экспериментальные данные. Штрих-пунктирная линия частота MER, $f_{\text {MER }}(H)$.

$(\omega / 2 \pi, k)=\left(\omega_{c} / 2 \pi, k_{c}\right), f_{\mathrm{MER}}(H)=\omega_{c} / 2 \pi$ при следующих значениях эффективной намагниченности и обменного поля: $4 \pi M_{\text {eff }} \sim 950 \mathrm{G}$ и $H_{\text {eff }}\left(k_{c}\right)=H+H_{\text {ex }}$, где $H_{\mathrm{ex}}=D k_{c}^{2} \sim 14 \mathrm{Oe}$.

Аналогичные зависимости резонансных частот были рассчитаны на основе выражения для электрического импеданса (14) с учетом подстановок (9)-(13). Магнитные и упругие потери при распространении волн во всех средах учитывались при помощи феноменологических параметров диссипации [19].

Численное решение полученное при подгоночном параметр $b=4.2 \cdot 10^{6} \mathrm{erg} / \mathrm{cm}^{3}$ показано на рис. 2 сплошными линиями. Как видно из экспериментальных данных, при заданном поле $H_{0}$ ширина магнитоупругой щели $\Delta f_{\text {MER }} \sim 30 \mathrm{MHz}$. В эту полосу попадает большое число резонансных частот $f_{n}$. Влияние MER на спектр резонатора постепенно спадает по мере удаления от линии $f_{\text {MER }}(H)$, однако остается заметным гораздо в более широкой области, чем $\Delta f_{\mathrm{MER}}$.
На рис. 3, $a$ приведен численный расчет частотной зависимости $\left|S_{11}\right|$ для поля 740 Ое. Частотная область влияния ADSWs на спектр локализована в точке максимального расталкивания дисперсионных ветвей $\operatorname{Re}\left[k_{1,2}(f)\right]$, (кривые 1,2$)$, приведенных на рис. 3, и и существенно превышает ширину MER, определяемую как $\Delta f_{\text {MER }}=\sqrt{\xi \omega_{H} \omega_{M}} / 2 \pi$. Решения для $\operatorname{Re}\left[k_{3}(f)\right] \ll \operatorname{Re}\left[k_{1,2}(f)\right]$ и $\operatorname{Im}\left[k_{3}(f)\right] \gg \operatorname{Im}\left[k_{1,2}(f)\right]$, лежат вне области диапазона, приведенного на рис. $3, d$.

В работе [21], сигнал напряжения $U_{\text {ISHE B }} \mathrm{Pt}$ измерялся в широком диапазоне частот возбуждения резонатора и записывался одновременно с частотными характеристиками HBAR. На рис. 4 показаны результаты такого одновременного сканирования при поле $H_{0}=744 \mathrm{Oe}$ [21], демонстрирующие резонансный характер по частоте $U_{\text {ISHE }}(f)$ с максимумами на частотах резонансов $f_{n}\left(H_{0}\right)$ (минимумы $\left|S_{11}\right|$ на рис. 4,a). Характерно, что максимальный сигнал $U_{\mathrm{ISHE}}(f)$ наблюдался не в центре MER, a ниже по частоте. Согласно оценке обменного поля, положение максимумов сигнала сдвинуто по частоте на величину обменного вклада в $f_{\text {MER }}\left(H_{0}\right)$, т.е.

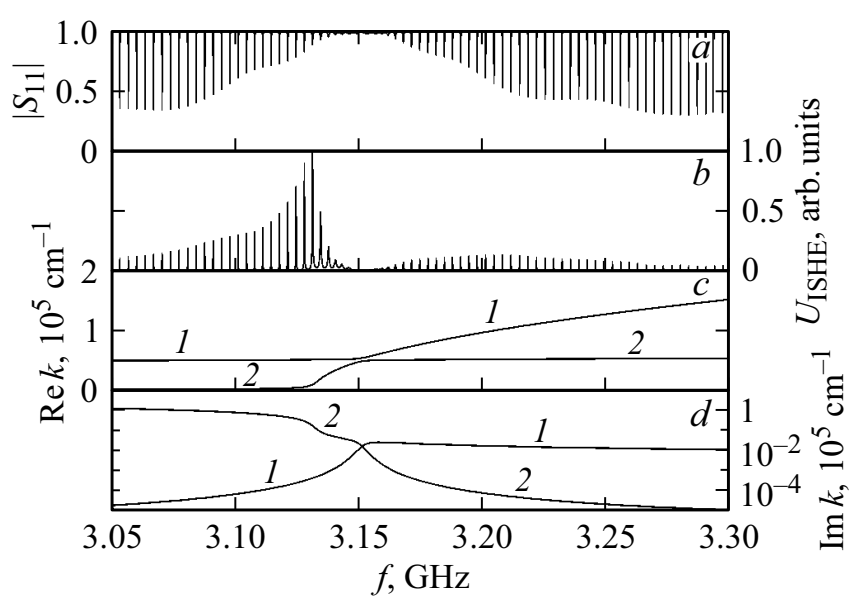

Рис. 3. Теоретические частотные зависимости: $a-$ модуля коэффициента отражения $\left|S_{11}\right|, b-$ сигнала постоянного напряжения, $c$ и $d-$ действительных и мнимых частей волновых чисел $k_{1}$ (кривые 1$)$ и $k_{2}$ (кривые 2) взаимодействующих мод.

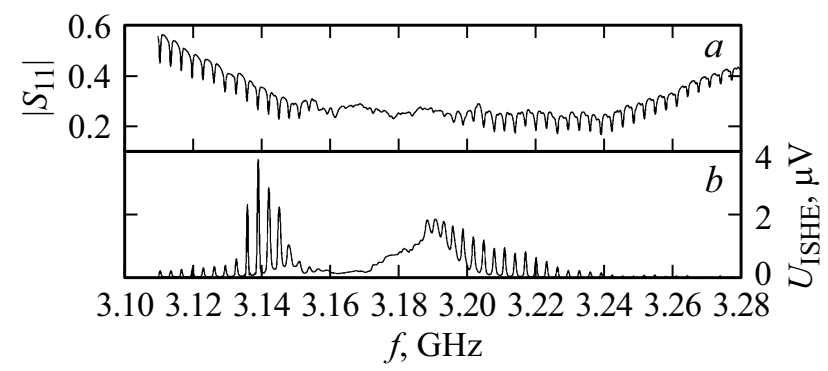

Рис. 4. Экспериментальные частотные зависимости модуля коэффициента отражения $\left|S_{11}\right| \quad(a)$ и постоянного напряжения на платине $(b)$ одновременно измеренные в поле $H_{0}=744$ Ое. Адаптировано из [21] (http://creativecommons.org./licenses/by/4.0/). 
локализовано вблизи дна зоны обменных SW. Это подтверждается также численными расчетами угла конуса прецессии (19) по полученным выше формулам для переменной намагниченности (15)-(18), приведенными на рис. $3, b$.

Принимая во внимание, что в расчете не учитывался ряд факторов, влиявших на эксперимент, в целом расчет дает ту же ассиметричную картину распределения максимумов относительно частоты $f_{\text {MER }}\left(H_{0}\right)$. Максимум огибающей $U_{\text {ISHE }}(f)$ проявляется вблизи частоты FMR $f_{\text {FMR }}\left(H_{0}\right)$, а сама огибающая смещена в область $f<f_{\text {FMR }}\left(H_{0}\right)$, т.е. в область эванесцентного решения для квазимагнонной дисперсии с мнимым волновым числом $k_{2}$. Эти решения, по-видимому, можно трактовать как поверхностные состояния, представляющие собой правополяризованное вращение вектора намагниченности, локализованное вблизи поверхности пленки YIG. Поскольку механизм генерации спинового тока также носит интерфейсный характер, то в платине скорее всего детектируются именно эта поверхностная намагниченность. Напротив, сдвиги резонансных частот обусловлены ADSWs во всем объеме пленки YIG и изменением фазовых соотношений из-за перестройки квазифононных законов дисперсии: ветви 1 при $f<f_{\text {MER }}\left(H_{0}\right)$ и ветви 2 при $f>f_{\text {MER }}\left(H_{0}\right)$ на рис. $3, c$.

\section{7. Заключение}

Получены аналитические выражения для электрического импеданса композитного СВЧ-резонатора объемных акустических волн со структурой $\mathrm{ZnO}-\mathrm{YIG}-\mathrm{GGG}-$ YIG-Pt, учитывающее магнитоупругую связь в слоях YIG и влияние наоднородного обмена на формирование спектра связанных волн. Развит теоретический подход для описания акустической спиновой накачки в резонаторе. Сравнение теоретических и экспериментальных магнитополевых зависимостей резонансных частот позволяет определить ряд магнитных параметров магнитных пленок. Теоретический расчет частотной зависимости напряжения ISHE показывает качественное соответствие экспериментальной зависимости [21] и объясняет ассимметричный характер поведения напряжения относительно частоты MER.

\section{Список литературы}

[1] Е.А. Туров, Ю.П. Ирхин. ФММ 3, 15 (1956).

[2] C. Kittel. Phys. Rev. 110, 836 (1958).

[3] А.И. Ахиезер, В.Г. Барьяхтар, С.В. Пелетминский. ЖЭТФ 35, 228 (1958).

[4] B. Flebus, K. Shen, T. Kikkawa, K. Uchida, Z. Qiu, E. Saitoh, R.A. Duine, G.E.W. Bauer. Phys. Rev B 95, 144420 (2017).

[5] M. Weiler, H. Huebl, F.S. Goerg, F.D. Czeschka, R. Gross, S.T.B. Goennenwein. Phys. Rev. Lett. 108, 176601 (2012).

[6] K. Uchida, H. Adachi, T. An, T. Ota, M. Toda, B. Hillebrands, S. Maekawa, E. Saitoh. Nature Mater. 10, 737 (2011).
[7] K. Uchida, T. An, Y. Kajiwara, M. Toda, E. Saitoh. Appl. Phys. Lett. 99, 212501 (2011).

[8] K. Uchida, H. Adachi, T. An, H. Nakayama, M. Toda, B. Hillebrands, S. Maekawa, E. Saitoh. J. Appl, Phys. 111, 053903 (2012).

[9] K. Uchida, Z. Qiu, T. Kikkawa, E. Saitoh. Solid State Commun. 198, 26 (2014).

[10] L. Dreher, M. Weiler, M. Pernpeintner, H. Huebl, R. Gross, M.S. Brandt, S.T.B. Goennenwein. Phys. Rev. B 86, 134415 (2012).

[11] D. Labanowski, A. Jung, S. Salahuddin. Appl. Phys. Lett. 108, 022905 (2016).

[12] S. Bhuktare, A. Bose, H. Singh, A.A. Tulapurkar. Sci. Rep. 7, 840 (2017)

[13] L. Thevenard, I.S. Camara, S. Majrab, M. Bernard, P. Rovillain, A. Lemaitre, C. Gourdon, J.-Y. Duquesne. Phys. Rev. B 93, 134430 (2016).

[14] P.G. Gowtham, T. Moriyama, D.C. Ralph, R.A. Buhrman. J. Appl. Phys. 118, 233910 (2015).

[15] N.I. Polzikova, S.G. Alekseev, I.I. Pyataikin, I.M. Kotelyanskii, V.A. Luzanov, A.P. Orlov. AIP Adv. 6, 056306 (2016).

[16] N.I. Polzikova, I.I. Pyataikin, S.G. Alekseev, I.M. Kotelyanskii, V.A. Luzanov, A.O. Raevskiy. In: Proc. 2016 IEEE Int. Frequency Control Symp. Piscataway. IEEE 372 (2016).

[17] N. Polzikova, S. Alekseev, I. Kotelyanskii, A. Raevskiy. In: Proc. 2013 IEEE Int. Ultrasonics Symp. Piscataway. IEEE 216 (2013).

[18] N. Polzikova, S. Alekseev, I. Kotelyanskii, A. Raevskiy, Yu. Fetisov. J. Appl. Phys. 113, 17C704 (2013).

[19] Н.И. Ползикова, А.О. Раевский, А.С. Горемыкина. Радиотехника и электроника 58, 97 (2013).

[20] E. Saitoh, M. Ueda, H. Miyajima, G. Tatara. Appl. Phys. Lett. 88, 182509 (2006).

[21] N.I. Polzikova, S.G. Alekseev, I.I. Pyataikin, V.A. Luzanov, A.O. Raevskiy, V.A. Kotov. AIP Adv. 8, 056128 (2018).

[22] A. Kamra, H. Keshtgar, P. Yan, G.E.W. Bauer. Phys. Rev. B 91, 104409 (2015).

[23] Н.М. Саланский, М.Ш. Ерухимов Физические свойства и применение магнитных пленок. Наука, Новосибирск (1975). $224 \mathrm{c}$.

[24] H.F. Tiersten. J. Appl. Phys. 36, 2250 (1965).

[25] Г. Кайно. Акустические волны. Устройства, визуализация, и аналоговоая обработка сигналов. Мир, М. (1990). 656 с.

[26] Э. Дьелесан, Д. Руайе. Упругие волны в твердых телах. Применение для обработки сигналов. Наука, М. (1982). $424 \mathrm{c}$.

[27] Y. Tserkovnyak, A. Brataas, G.E. Bauer. Phys. Rev. Lett. 88, 117601 (2002).

[28] M. Schreier, G.E.W. Bauer, V.I. Vasyuchka, J. Flipse, K. Uchida, J. Lotze, V. Lauer, A.V. Chumak, A.A. Serga, S. Daimon. J. Phys. C 48, 025001 (2015).

Редактор Ю.Э. Китаев 